\title{
Comparison of the conditioning of high gradient accelerating structures
}

\author{
Alberto Degiovanni ${ }^{\dagger}$ and Walter Wuensch* \\ CERN, European Organization for Nuclear Research, 1211 Geneva, Switzerland \\ Jorge Giner Navarro \\ Instituto de Física Corpuscular (CSIC-UV), E-46071 Valencia, Spain \\ (Received 3 June 2015; published 4 March 2016)
}

\begin{abstract}
Accelerating gradients in excess of $100 \mathrm{MV} / \mathrm{m}$, at very low breakdown rates, have been successfully achieved in numerous prototype CLIC accelerating structures. The conditioning and operational histories of several structures, tested at KEK and CERN, have been compared and there is clear evidence that the conditioning progresses with the number of $\mathrm{rf}$ pulses and not with the number of breakdowns. This observation opens the possibility that the optimum conditioning strategy, which minimizes the total number of breakdowns the structure is subject to without increasing conditioning time, may be to never exceed the breakdown rate target for operation. The result is also likely to have a strong impact on efforts to understand the physical mechanism underlying conditioning and may lead to preparation procedures which reduce conditioning time.
\end{abstract}

DOI: 10.1103/PhysRevAccelBeams.19.032001

\section{INTRODUCTION}

The CLIC accelerating gradient target [1] of $100 \mathrm{MV} / \mathrm{m}$, pulse length of around $200 \mathrm{~ns}$ and breakdown rate (BDR, i.e., number of pulses with breakdown divided by total number of pulses) in the range of $10^{-7} 1 / \mathrm{pulse} / \mathrm{m}$ is now routinely achieved in prototype CLIC accelerating structures [2]. The design, manufacture and testing of the prototypes is a well-established process. The limiting gradient behavior has been comprehensively studied and reasonably well understood [3]. In particular, a quantity based on local power flow [4] appears to determine the limiting gradient as a function of rf design for a given manufacturing procedure. Prototype structures are manufactured reproducibly by KEK/SLAC and CERN according to a procedure developed in the NLC/GLC linear collider study [5]. Relevant details of the manufacturing procedure will be summarized below. The structures discussed in this paper have been tested at KEK and CERN in klystronpowered test stands [6,7]. Testing is done almost entirely under computer control, and the underlying algorithm allows the structures to be conditioned while maintaining at a user-selected breakdown rate.

The ultimate performance of the structures is now acceptable for the CLIC project; however all the structures tested so far have required a lengthy, three to four month, $\mathrm{rf}$ conditioning period to arrive to the target performance.

\footnotetext{
*walter.wuensch@cern.ch

Present address: A.D.A.M. SA, 1209 Geneva, Switzerland.
}

Published by the American Physical Society under the terms of the Creative Commons Attribution 3.0 License. Further distribution of this work must maintain attribution to the author(s) and the published article's title, journal citation, and DOI.
Since such a long conditioning period would represent a significant contribution to the total cost of the structures, reducing the conditioning time is an important priority for the CLIC study. A dedicated effort to understand and reduce $\mathrm{rf}$ conditioning time has been initiated and the first step in this effort has been to rigorously compare the conditioning data from a set of similar rf design prototype CLIC accelerating structures. This analysis has surprisingly produced clear evidence that the conditioning progresses as the number of rf pulses and not as the number of breakdowns. This analysis is described in this paper.

The insight that conditioning progresses with the number of pulses, rather than with the number of breakdowns, points towards an improved conditioning procedure. The number of breakdowns a structure is exposed to can be minimized, without increasing the conditioning time compared to current procedures, by never exceeding the target operational breakdown rate, which is $3 \times 10^{-7} 1 / \mathrm{pulse} / \mathrm{m}$ in the case of CLIC. Minimizing the number of breakdowns could limit damage to the structure and the appearance of so-called "hot" cells. In addition the insight will be used as input for the investigation of new structure preparation procedures to reduce the rf conditioning time by mimicking the effect of rf pulses in a cost effective way.

\section{EXPERIMENTAL SETUP AND OPERATIONAL ALGORITHMS}

Since the rf design of an accelerating structure has a very strong influence on rf performance [1], this comparative conditioning analysis has been done on the largest subgroup of similar CLIC prototype structures which have been tested so far. The structures considered here are the so-called TD26CC and TD24R05 (please see [1] for 
nomenclature). These two types of structures have identical main cells but the input and output power couplers have different $\mathrm{rf}$ designs. The main fabrication features and procedures of the structures are the same: 4. OFE (OxygenFree Electronic copper) copper, diamond-turned cells, hydrogen bonding and vacuum bakeout. CERN-tested structures were fabricated by CERN and KEK-tested structures were made jointly by KEK and SLAC. The rf frequency was $11.994 \mathrm{GHz}$ at CERN and $11.424 \mathrm{GHz}$ at KEK. Details of the design and fabrication of these structures can be found in [1].

The structures were tested in either the CERN Xbox-1 facility [7] or the KEK Nextef facility [8]. Xbox-1 is a $12 \mathrm{GHz}$ high power klystron test stand dedicated primarily to the test of CLIC prototype accelerating structures. The high-power network of the test stand consists of a $50 \mathrm{MW}$ klystron, a solid state modulator and pulse compressor. Further information on Xbox-1 can be found in [7]. The Nextef test stand at KEK was constructed during the NLC/ GLC project. It has been operated in recent years to support the CLIC accelerating structure development program. It consists of two $50 \mathrm{MW}$ klystrons which are combined. High-gradient tests made in Nextef are reported in [6] and references therein.

The testing protocols at the two test facilities are similar. Breakdowns in both facilities are detected by a combination of measurement of current bursts via Faraday cups and measurement of increased reflected rf power. High-gradient operation in Xbox-1 is controlled by an automated conditioning algorithm with operator intervention necessary only to set a few global parameters like pulse length and target breakdown rate. The essential features of the algorithm include a loop for recovery from a breakdown and power ramping for conditioning. Loop parameters are automatically revised in a slow feedback loop to ensure conditioning occurs at the target breakdown rate. In Xbox1 , an rf pulse compressor was used during the tests described here and the conditioning system was always operated at $50 \mathrm{~Hz}$. More details of breakdown detection and the operational algorithm can be found in [7]. The Nextef conditioning control is somewhat less automated. The conditioning rate in Nextef is the outcome of user-defined power ramping rate which is typically updated daily to maintain the target value.

Accelerating structures in both test stands, as well as previous testing at SLAC in NLCTA [9], are also ramped up in pulse length as the conditioning state of the structure progresses. Conditioning starts with a 50 to $70 \mathrm{~ns}$ pulse at low power. The power is gradually increased by the conditioning algorithm, while maintaining the target breakdown rate, until the target gradient is reached. At this point the power is reduced by about $20 \%$, the pulse is lengthened and the power ramp begins again. This is repeated for about five pulse lengths until the final target pulse parameters have been achieved. The choice of pulse length and of pulse length steps are set by the operator in both Xbox-1 and Nextef. The typical overall testing time for the structures described here is about six months.

\section{DATA AND ANALYSIS}

The upper plot of Fig. 1 shows the achieved accelerating gradient (green), the pulse length during each period of operation (black text) and the accumulated number of breakdowns (red) with respect to the accumulated number of pulses of a TD26CC structure tested at CERN. The slope of the red curve, fit over an appropriate number of pulses to average out fluctuations, gives the local breakdown rate. The most important parameters used during the conditioning of the structure are summarized in Table I.

In addition to the increase in the pulse length from period 1 to period 2, the target $\mathrm{BDR}$ was lowered to $2 \times 10^{-5} 1 /$ pulse (from about $7 \times 10^{-5} 1 /$ pulse). This was to avoid the appearance of "sawtoothing"-the situation when the conditioning algorithm would ramp the power, apparently too quickly, causing the structure to break down repeatedly which in turn caused the algorithm to react by significantly lowering the power in order to reestablish the target BDR. This condition was considered potentially risky for the structure and could be eliminated by simply reducing the target breakdown rate. More details on the testing of the TD26CC structure can be found in [2]. The active conditioning period, when power and pulse length were ramped to maintain a target breakdown rate, ended when the gradient and pulse length were $105 \mathrm{MV} / \mathrm{m}$ and $250 \mathrm{~ns}$ (flattop) respectively. This took about 1650 hours of testing time (equivalent to four months of operation with an overall up-time of about 65\%). The active conditioning period was followed by a run with constant input power conditions, $100 \mathrm{MV} / \mathrm{m}$ and $250 \mathrm{~ns}$. The breakdown rate was free to change and decreased steadily.

The operational history of a TD24R05 structure tested at KEK is shown in the lower part of Fig. 1. The overall characteristics of the structure conditioning are similar, however the pulse lengths were different and changes in pulse length were made at different times. In addition the KEK active conditioning period was followed by a much longer run with constant input conditions, which were changed from time to time during measurements of the dependence of BDR on gradient and pulse length. Almost all breakdowns occurred in the main cells of the structures which have been tested, which means that the results can be compared even though the input and output power coupler designs in the CERN-tested TD26CC and KEK tested TD24R05 are different, as mentioned in Sec. II.

In order to directly compare the behavior of the two structures throughout their operating history, the data must be scaled to account for the differing operating conditions (gradient, pulse length and BDR) in the two cases. This can be done using dependencies of breakdown rate on gradient 

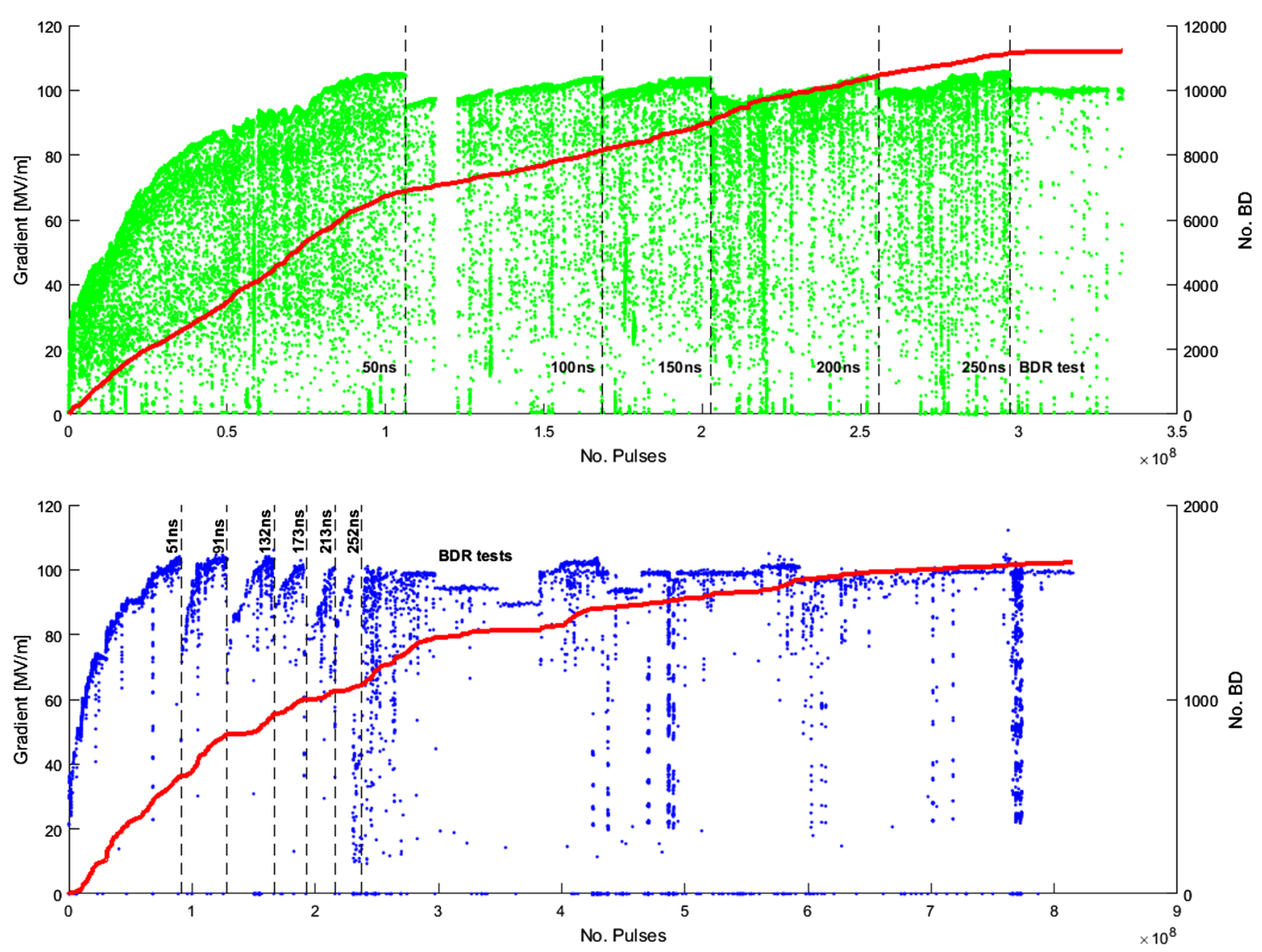

FIG. 1. Summary of CERN TD26CC and KEK TD24R05\#4 raw data. The green and blue dots represent the unloaded gradient (left axis) excited into the structure as a function of the number of pulses. The red solid line indicates the cumulated number of breakdowns (right axis). The dots that fall below the envelope are due to the power ramping applied after a breakdown event. For the rest of the analysis these points have been discarded. The changes in pulse widths are indicated by vertical lines.

and pulse length, which have been observed in experiments and are reported in [4]. The dependencies can be approximated with the relation,

$$
\frac{E_{0}^{30} \cdot \tau^{5}}{\mathrm{BDR}}=\mathrm{const}
$$

where $E_{0}$ indicates the accelerating gradient and $\tau$ the $\mathrm{rf}$ pulse length. Based on this scaling law, we rearrange terms and define the "scaled gradient":

$$
E_{0}^{*}=\frac{E_{0} \cdot \tau^{1 / 6}}{\mathrm{BDR}^{1 / 30}}
$$

TABLE I. Main operational parameters during the conditioning of the TD26CC structure at CERN.

\begin{tabular}{lcccc}
\hline \hline Period & $\tau[\mathrm{ns}]$ & $E_{0}[\mathrm{MV} / \mathrm{m}]$ & BDR $[1 /$ pulse $]$ & Time $[\mathrm{hrs}]$ \\
\hline 1 & 50 & $30-110$ & $7 \times 10^{-5}$ & 580 \\
2 & 100 & $90-110$ & $2 \times 10^{-5}$ & 360 \\
3 & 150 & $95-110$ & $2 \times 10^{-5}$ & 200 \\
4 & 200 & $95-110$ & $2 \times 10^{-5}$ & 290 \\
5 & 250 & $95-105$ & $2 \times 10^{-5}$ & 220 \\
\hline \hline
\end{tabular}

Equation (2) has been applied to the CERN TD26CC data and the result is shown in Fig. 2. What emerges is a rather smooth curve which increases steadily over the course of the experiment and is no longer segmented by the different operating conditions. The smoothness of the scaled gradient curve indicates that the quantity represents a well-defined physical state of the structure, and the steady rise that it represents the conditioning state of a structure. The scaled gradient also allows a direct comparison of different structures. These topics will be addressed in the following section.

\section{CONDITIONING COMPARISON}

The scaled gradient can now be used to directly compare operational data from different structures so that, for example, the conditioning rate can be quantitatively compared independently of the particular operational parameters of the experiment. The scaled gradient as a function of operating time has been compared for four similar structures (described in Sec. II): a TD26CC tested in Xbox-1, a TD24R05 tested in Xbox-1 and two TD24R05s tested at Nextef. The first type of comparison, shown in Fig. 3, is a 


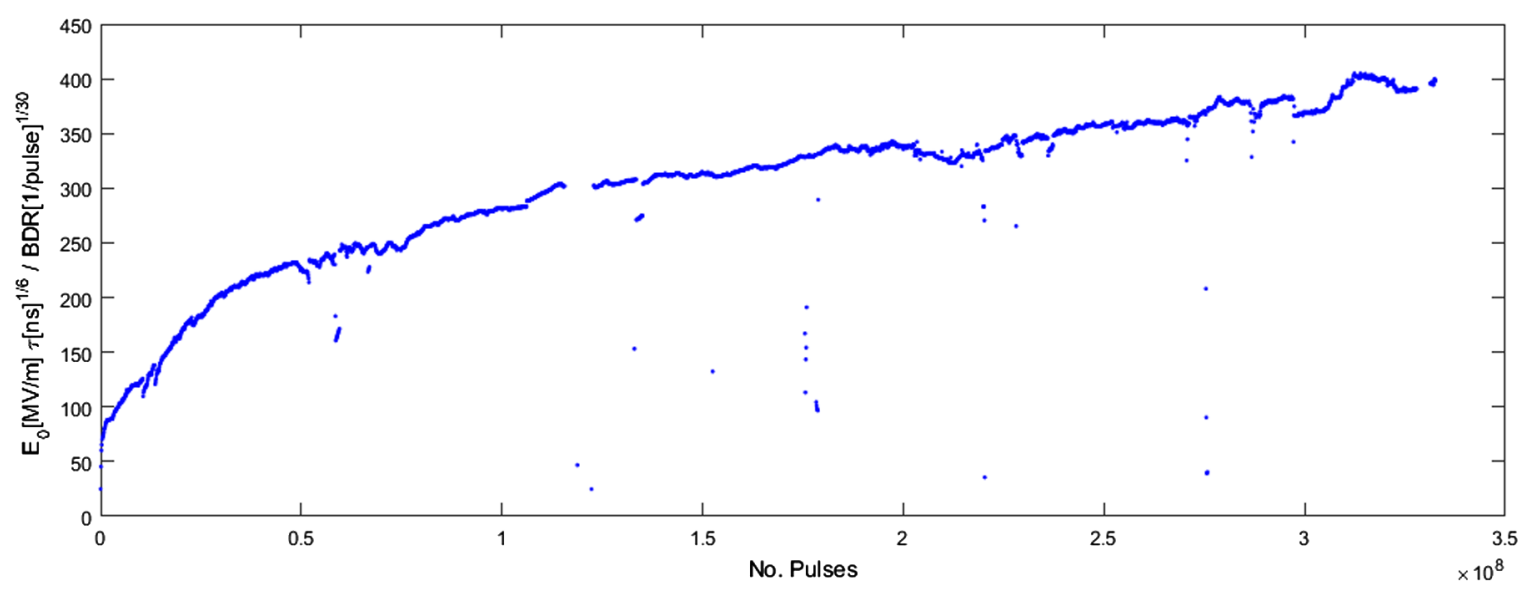

FIG. 2. Scaled gradient vs number of accumulated pulses for the structure TD26CC tested at CERN. The rescaling has been done applying the definition in Eq. (2).

plot of the scaled gradient against the number of pulses. The conditioning behavior of the four structures is remarkably similar. The second type of comparison, shown in Fig. 4, is a plot of the scaled gradient versus the number of accumulated breakdowns. In this case there is a significant divergence in the behavior of the four structures. This probably reflects the different target breakdown rate (selected through the values of the parameters of the operational algorithm) used at the two test areas. The Xbox-1 target rate was roughly a factor of 5 to 10 higher than the Nextef rate, and this is reflected in the accumulated number of breakdowns to reach the same scaled gradient.

The stronger correlation of scaled gradient as a function of the number of pulses compared to the number of breakdowns indicates that conditioning progresses with the number of accumulated rf pulses and not with the number of accumulated breakdowns.

In other words, breakdowns occur during conditioning but they are not the cause of it. This observation can be used to optimize the operation of high-gradient accelerating structures, and in a way based on clear experimental evidence. For the time being this observation has only been tested on a limited group of structures, however subsequent structures at the Xboxes and Nextef will be compared in the same way. Determining whether or not the observation is valid beyond high-gradient X-band structures will require appropriate quantitative operational data from other types of normal conducting rf structures.

The observation that conditioning progresses with the number of pulses rather than the number of breakdowns leads to a number of specific consequences. The first is that conditioning at a low breakdown rate does not take longer than at high breakdown rate, and certainly reduces the risk of accumulated damage to the structure by limiting the number of breakdowns, so is a better strategy. It may be that conditioning rate depends on the field level but there is no evidence of this in the data considered here. The effectiveness of the low breakdown rate conditioning strategy will be further tested in the test stands and in the CERN dc spark system [10].

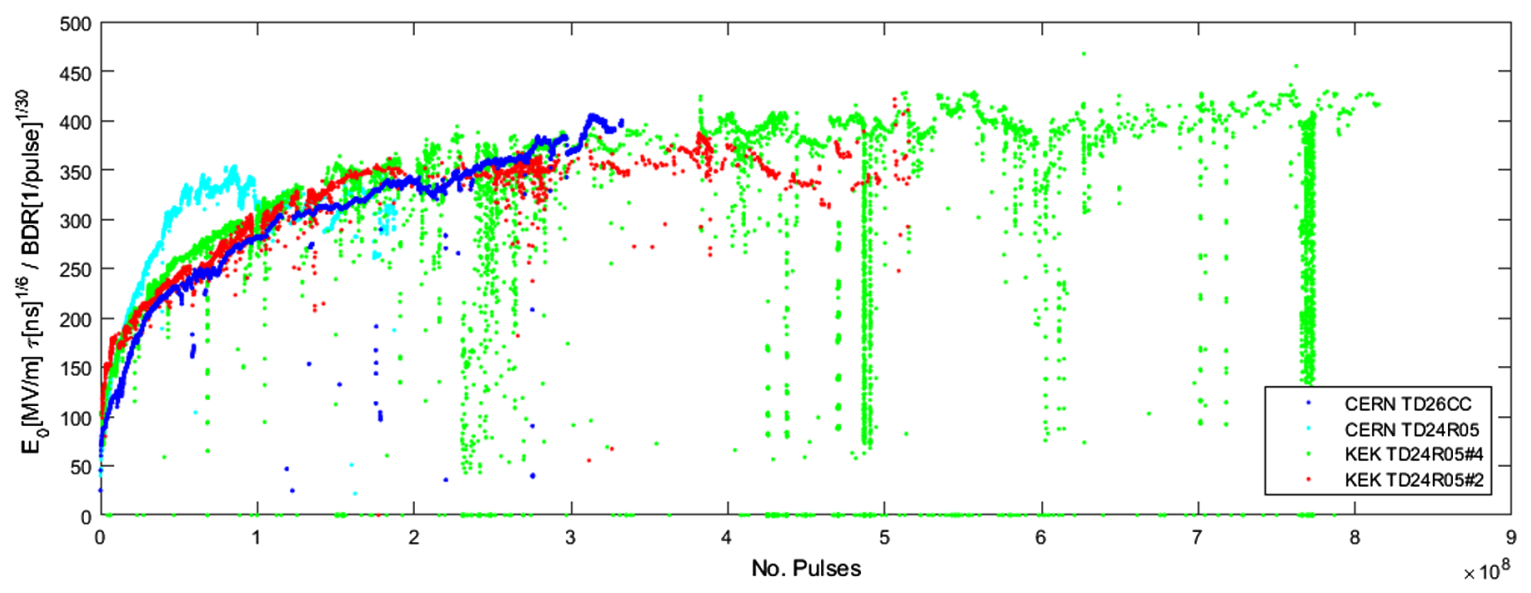

FIG. 3. Comparison of the scaled gradient vs number of accumulated pulses for several structures. Despite the different conditioning approaches, the curves for the scaled gradient are similar. 


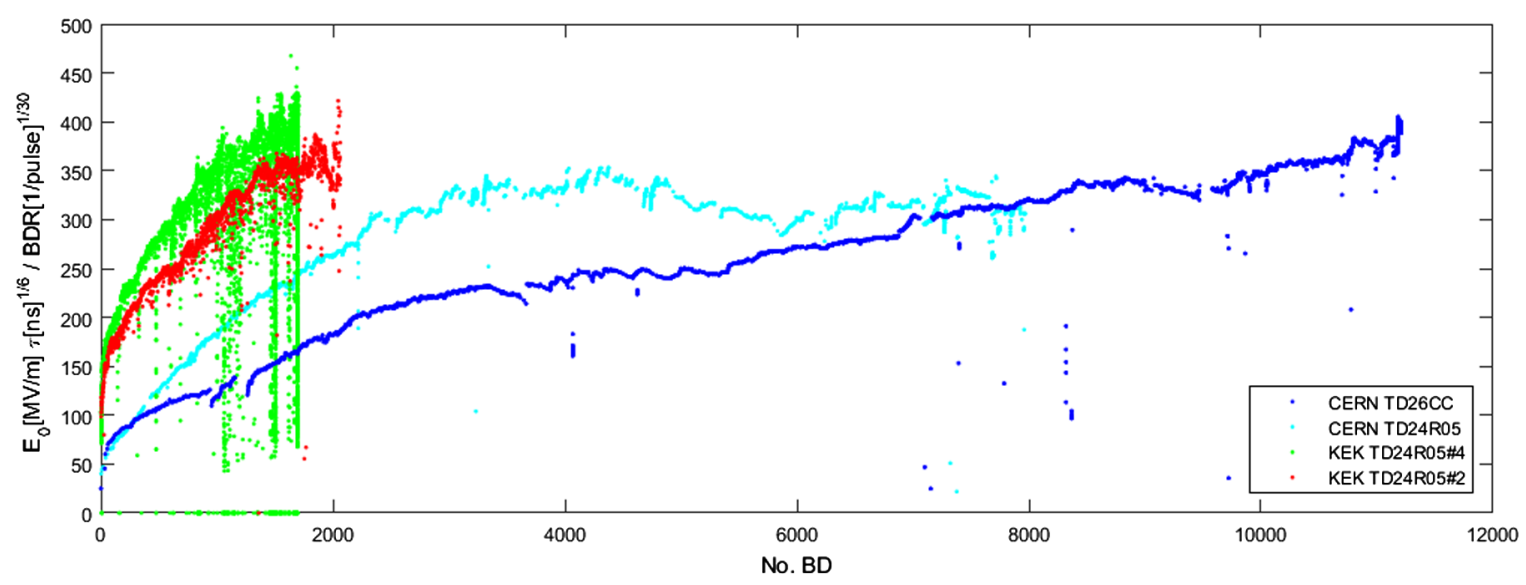

FIG. 4. Comparison of the scaled gradient vs number of accumulated breakdowns for several structures. When plotted with respect to the total accumulated number of breakdowns, the curves of the scaled gradient diverge significantly.

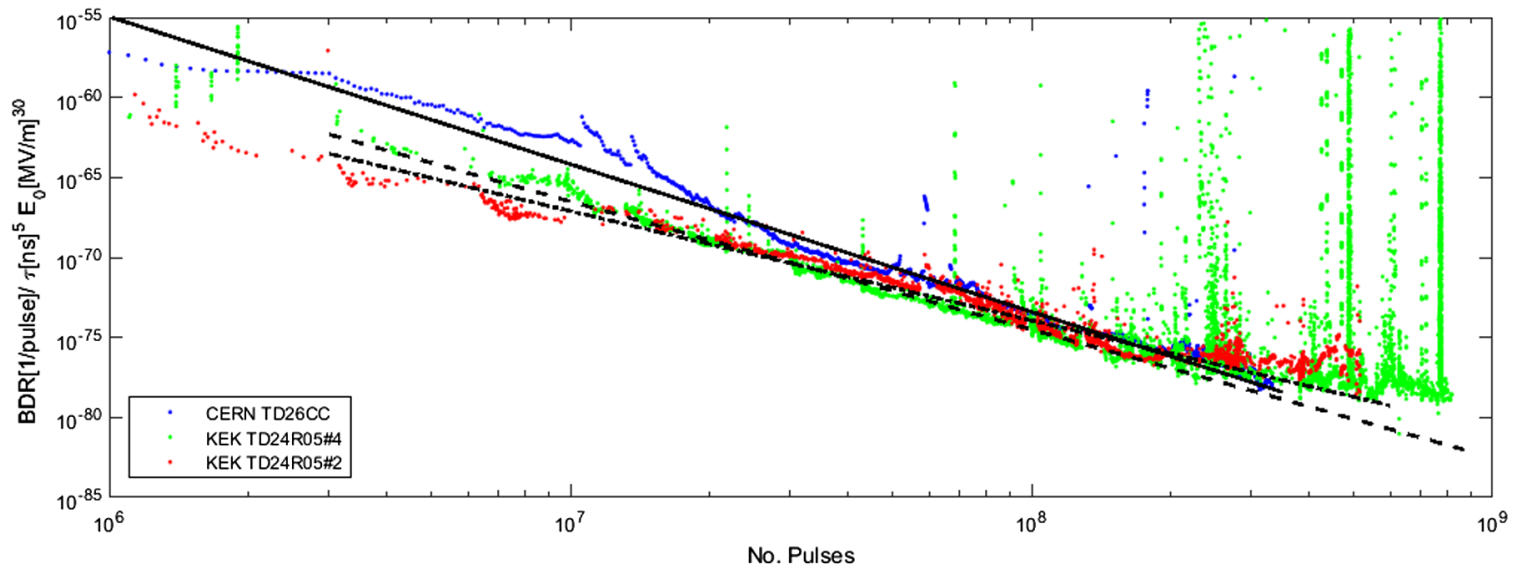

FIG. 5. Comparison of scaled BDR for different structures. The data are plotted in a log-log scale. The scaled BDR is decreasing monotonically with respect to the number of pulses. The curves are fitted with a power law.

The second consequence is that each rf pulse appears to act to modify the structure surface to strengthen it against high fields and/or reduce or eliminate potential breakdown sites in a way which does not result in a fully evolved and detectable breakdown event. Identifying the relevant strengthening mechanisms would be particularly valuable since it would open the possibility of developing, in a targeted way, a surface treatment procedure which reproduces the effect of $\mathrm{rf}$ conditioning but at a lower cost.

This same data analysis can also be used to evaluate when a structure is approaching its highest performance. In this case it is helpful to plot the scaled breakdown rate, which can be found by rewriting Eq. (1) as follows:

$$
\mathrm{BDR}^{*}=\frac{\mathrm{BDR}}{E_{0}^{30} \cdot \tau^{5}}
$$

The result is shown in Fig. 5. By comparing Eq. (2) with Eq. (3) the following relation holds:

$$
\mathrm{BDR}^{*}=1 / E_{0}^{* 30} .
$$

The conditioning can be fit very well with a power law of the type

$$
\mathrm{BDR}^{*}=C \times n_{p}^{A},
$$

where $n_{p}$ is the number of pulses and with exponents A summarized in Table II.

TABLE II. Summary of power law fit for the scaled breakdown rate of the structures under study.

\begin{tabular}{lc}
\hline \hline Structure name & A \\
\hline TD26CC & -9.2 \\
TD24R05\#2 & -6.8 \\
TD24R05\#4 & -8.0 \\
\hline \hline
\end{tabular}


One can see divergences from the power law fit which may indicate that the conditioning state of a structures is no longer improving. The structure CERN TD24R05 has not been included in the analysis of long-term behavior since it developed a hot cell, a jump in the breakdown rate in a single cell, at about $10^{8}$ pulses. This feature can be seen in Fig. 3 and is believed to be due to too aggressive, too high a target breakdown rate, conditioning parameters used at the time.

The power law fit of the conditioning in principle also potentially gives an insight into the nature of the conditioning mechanism.

\section{CONCLUSION}

By using established scaling laws, operational data can be reanalyzed with the result that high-gradient rf structures show a smooth, continuous progression in their conditioning state, despite varying operating conditions.

A comparison of the conditioning behaviors of four similar high-gradient accelerating structures tested at CERN and at KEK suggests that the conditioning state improves with the number of $\mathrm{rf}$ pulses and not with the number of breakdowns.

These insights have important ramifications for optimizing conditioning strategies, understanding the mechanism of conditioning and for potentially developing preparation techniques which reduce conditioning time and consequently overall investment cost.

\section{ACKNOWLEDGMENTS}

The authors would like to sincerely thank the structure production, CERN Xbox and KEK Nextef teams for their enormous efforts in preparing and carrying out the experiments on which this analysis is based. We would also like to thank personally Alexej Grudiev and Igor Syratchev for many useful discussions while these ideas were being formed. A special thank goes to Ben Woolley and Joseph Tagg for the development of the automatic conditioning software in Xbox-1, a crucial development which made this analysis possible.
[1] M. Aicheler, P. Burrows, M. Draper, T. Garvey, P. Lebrun, K. Peach, N. Phinney, H. Schmickler, D. Schulte, and N. Toge, A multi-TeV linear collider based on CLIC technology, CLIC Conceptual Design Report, CERN 007, 2012.

[2] A. Degiovanni, S. Doebert, W. Farabolini, A. Grudiev, J. Kovermann, E. Montesinos, G. Riddone, I. Syratchev, R. Wegner, W. Wuensch, A. Solodko, and B. Woolley, High-Gradient Test Results from a CLIC Prototype Accelerating Structure: TD26CC, in Proceedings of the International Particle Accelerator Conference IPAC14 (2014), pp. 2285-2287.

[3] W. Wuensch, Advances in the understanding of the physical processes of vacuum breakdown, Report No. OPEN-2014028; Report No. CLIC-Note-1025, CERN, 2014.

[4] A. Grudiev, S. Calatroni, and W. Wuensch, Phys. Rev. ST Accel. Beams 12, 102001 (2009).

[5] J. W. Wang, J. R. Lewandowski, J. W. V. Pelt, C. Yoneda, G. Riddone, D. Gudkov, T. Higo, and T. Takatomi, in Proceedings of the International Particle Accelerator Conference, Kyoto, Japan (ICR, Kyoto, 2010), pp. 3819-3821.

[6] T. Higo, T. Abe, Y. Arakida, S. Matsumoto, T. Shidara, M. Y. T. Takatomi, A. Grudiev, G. Riddone, and W. Wuensch, in Proceedings of the 4th International Particle Accelerator Conference, IPAC-2013, Shanghai, China, 2013 (JACoW, Shanghai, China, 2013), pp. 2741-2743.

[7] N. Catalan-Lasheras, A. Degiovanni, S. Doebert, W. Farabolini, J. Kovermann, G. McMonagle, S. Rey, I. Syratchev, L. Timeo, W. Wuensch, B. Woolley, and J. Tagg, Experience Operating an X-Band High-Power Test Stand at CERN, in Proceedings of International Particle Accelerator Conference IPAC14 (2014), pp. 2288-2290.

[8] S. Matsumoto, T. Abe, Y. Higashi, T. Higo, and Y. Du, Nucl. Instrum. Methods Phys. Res., Sect. A 657, 160 (2011).

[9] C. Adolphsen, G. Bowden, V. Dolgashev, L. Laurent, S. Tantawi, F. Wang, J. Wang, S. Doebert, A. Grudiev, G. Riddone, W. Wuensch, R. Zennaro, Y. Higashi, and T. Higo, in Proceedings of the 23rd Particle Accelerator Conference, Vancouver, Canada, 2009 (IEEE, Piscataway, NJ, 2009), pp. 2027-2029.

[10] N. C. Shipman, Experimental study of dc vacuum breakdown and application to high-gradient accelerating structures for CLIC, Ph.D. thesis, University of Manchester, 2014. 